\title{
Editorial
}

\section{Nanoparticle Enhanced Imaging}

\author{
Mukesh G. Harisinghani* \\ Harvard Medical School, Director Abdominal MRI, Massachusetts General Hospital, Boston, MA 02114, USA
}

\begin{abstract}
Nanotechnology refers to the research and technical development of objects in a scale range of 1 to 100 nanometers. Particles that are nano-sized are opening new research avenues in the field of engineering and life sciences. Imaging with biocompatible nanoparticles has emerged as one of the most promising new diagnostic imaging technology fulfilling the goal of developing cost-effective magnetic nanoparticles with acceptable targeting efficiency, favorable pharmacokinetics and a good safety profile. These nanoparticles target the reticuloendothelial system and this combined with unique properties of superparamagnetism allows them to be used as excellent probes for magnetic resonance imaging (MRI). The current issue focusses on the use of a specific type of magnetic nanoparticle (ferumoxtran10) to overcome imaging handicaps in cancer staging as well as discuss emerging applications of newer magnetic nanoparticles. Imaging with ferumoxtran-10 offers the potential of staging tumors at stages much earlier that those detectable today.

Therapy decisions and prognostic predictions for most tumors depend on the accurate lymph node staging at the time of initial diagnosis. Despite the importance of nodal status, accurate staging of lymph nodes in various primary tumors continues to pose a major diagnostic challenge. Following the detection of lymph nodes, the next arduous task is the characterization of nodes into benign and malignant categories. Current cross-sectional modalities like CT and MRI primarily rely on nodal size and nodal morphology for differen-
\end{abstract}

*Corresponding author. Tel.: +1 617726 8396; Fax: +1 617 726 4891; E-mail: mharisinghani@partners.org; mharisinghani @mgh.harvard.edu. tiating benign from malignant lymph nodes. In comparison, the emerging modality of PET relies on the metabolic activity for nodal characterization. Despite their widespread use for detecting and staging the primary tumors, cross sectional modalities are limited in their ability to characterize lymph nodes as there is considerable overlap in the imaging features of benign and malignant lymph nodes. Using FDG-PET overcomes some of these disadvantages. The ability of PET to detect malignant nodes is however largely dependent on the FDG avidity of the primary tumor and also on the size of the affected node. Primary prostate cancer for example is not very 18-FDG avid, and hence PET cannot be reliably used for nodal staging and detection of small malignant nodes. Herein lies the emerging role of nanoparticle or ferumoxtran-10 enhanced MRI also called lymphotrophic nanoparticle enhanced MRI (LNMRI). Several published studies have shown that LNMRI can overcome the imaging handicaps by targeting and binding to macrophages within normal lymph nodes. This binding property combined with the distinct MRI signature of magnetic nanoparticles allows easy identification of normal sized nodes in the expected distribution for various primary cancers. The lack of nanoparticle uptake even in small sized nodes or small nodal regions infiltrated with tumor allows accurate distinction between benign and malignant lymph nodes independent of the size of affected lymph node. The ensuing articles provide an overview of the mechanism and pharmacokinetics of ferumoxtran-10 as well as elucidate the efficacy or LNMRI using ferumoxtran10 in various primary malignancies. This is followed by a brief overview of next generation nanoparticles that further enhance accuracy and ease of use. 